\title{
Dynamic analysis of a pendulum dynamic automatic balancer
}

\author{
Jin-Seung Sohn ${ }^{\mathrm{a}}$, Jin Woo Lee ${ }^{\mathrm{b}}$, Eun-Hyoung Cho ${ }^{\mathrm{a}}$, No-Cheol Park ${ }^{\mathrm{c}, *}$ and Young-Pil Park ${ }^{\mathrm{b}}$ \\ a Storage Laboratory Samsung Advanced Institute of Technology, P.O. Box 111, Suwon 440-600, Korea \\ ${ }^{\mathrm{b}}$ Department of Mechanical Engineering, Yonsei University, 134 Shinchon-dong, Sudaemun-ku, Seoul 120-749, \\ Korea \\ ${ }^{\mathrm{c}}$ Center for Information Storage Device, Yonsei University, 134 Shinchon-dong, Sudaemun-ku, Seoul 120-749, \\ Korea
}

Received 4 June 2004

Revised 16 May 2005

\begin{abstract}
The automatic dynamic balancer is a device to reduce the vibration from unbalanced mass of rotors. Instead of considering prevailing ball automatic dynamic balancer, pendulum automatic dynamic balancer is analyzed. For the analysis of dynamic stability and behavior, the nonlinear equations of motion for a system are derived with respect to polar coordinates by the Lagrange's equations. The perturbation method is applied to investigate the dynamic behavior of the system around the equilibrium position. Based on the linearized equations, the dynamic stability of the system around the equilibrium positions is investigated by the eigenvalue analysis.

The stability analysis provides the design requirements for the pendulum automatic dynamic balancer to achieve a balancing of the system. The efficiency of ball automatic dynamic balancer, for reducing the total vibration of the system, is better than one of pendulum automatic balancer, if the rotating speed is above critical speed. However, pendulum automatic dynamic balancer can achieve balancing even if the rotating speed is below critical speed.

The time response analysis demonstrates the stability analysis from computing the radial displacement of the rotating system and the positions of pendulums. Furthermore, in order to confirm the theoretical analysis, various experiments are made on pendulum automatic dynamic balancer.
\end{abstract}

\section{Introduction}

Many researchers have focused on reducing variable unbalance and vibratory force on the rotating system during normal operation. The research has led to automatic dynamic balancer, usually composed of a circular disk with a race, containing spherical weights and a low viscosity damping fluid. The concept of an automatic dynamic balancer is applicable to various rotating machinery which are subject to have variable unbalances.

The automatic dynamic balancer has been proposed in many patents to minimize the effects of rotor unbalance and vibratory forces on the rotating system. Alexander presented the result of a theoretical analysis of automatic dynamic balancer concept [1]. The configuration consisted of counterweights, free to rotate in races, located in a long, slender and dynamically unbalanced rotating body. However it was not stated how the counterweights move and how this motion is related to that of the long and slender body. Cade suggested the requirements for automatic dynamic balancer, but the theoretical background was not explained [2]. Lee also conducted theoretical and experimental analysis of an automatic dynamic balancer and showed that the automatic dynamic balancer could balance a rotating system when the system operated above the critical speed [3]. However, the reason why an automatic dynamic

*Corresponding author. Fax: +82 23658460 ; E-mail: pnch@yonsei.ac.kr. 
balancer could not balance the system, in some cases, was not explained even when an automatic dynamic balancer operated above the critical speed.

Recently, Chung analyzed the stability and dynamic behavior of an automatic dynamic balancer using the polar coordinate system instead of the rectangular system [4]. It was shown that the use of the polar coordinate system made it possible to derive the equations of motion for the autonomous system efficiently. The dynamic states of the system were divided into two classes; the stable regions and unstable regions. It is also shown that the fluid damping is helpful for the automatic dynamic balancer to achieve balancing. Hwang analyzed dynamic behavior of an automatic dynamic balancer with double races, and showed the possibility of fine balancing, avoiding the impact between the balls [5]. Cho analyzed dynamic behavior of an automatic dynamic balancer with triple races, showing that it could have various equilibrium positions [6]. Huang investigated the dynamic effects of runway eccentricity, rolling resistance and drag force on a rotor-balancer system that is designed to reduce residual unbalanced vibration [7]. Bae showed that the hydraulic balancer, using salt water as the working fluid, is more effective than a solid balancer, in the case of washing machine [8].

Various kinds of self-compensating dynamic balancers have been suggested, but most researches have been published and focused on automatic dynamic balancer using the weight of balls as the compensating unbalance. However for an automatic dynamic balancer using balls, which is named ball automatic dynamic balancer(BADB), it is difficult to secure the repeatability of balancing. It has been proven that the main reasons for the lack of repeatability is rolling friction between balls and race, and contact sliding friction between adjacent balls by Sohn [9]. Additionally, the collision of balls can have adverse effects upon dynamic stability.

An alternative automatic dynamic balancer which is composed of the weights, such as pendulums, suspended by pivots around center of rotating system instead of balls rolling inside of race; so called a pendulum automatic dynamic balancer (PADB) can substitute BADB because a PADB can solve some of the problems that a BADB has. Although a PADB also has friction on pivot point, it can avoid the friction between moving masses and as a result a PADB has much better repeatability than a BADB. Moreover, a PADB which is equipped with the pendulum motion limit stops that confine the angular movement of pendulums increases the dynamic stability by restricting excessive angular motion of pendulums and avoiding collision between pendulums.

Chung's research shows that a BADB can reduce the total vibration of the system, if the rotating speed is above suspension natural frequency, but the system cannot be balanced below suspension natural frequency [4].

In practical applications, multiple compensating masses are installed to the automatic dynamic balancer for the improvement of balancing performance. But in the numerical study of the automatic dynamic balancer dynamics, the number of compensating masses are minimized to avoid the complexity of analysis [3-7], The minimum number of pendulums for the compensation of arbitrarily unbalanced disk is two. In this paper, the stability and dynamic behavior of a PADB and a BADB are compared by theoretical and experimental analysis. First, for the analysis of dynamic stability and behavior, the nonlinear equations of motion for the PADB are derived with respect to the polar coordinate system by Lagrange's equation. Second, from the nonlinear equations, the equilibrium positions and the linearized equations are calculated by the perturbation method. Third, based on the linearized equations, the dynamic stability of the system around the equilibrium positions are investigated by the eigenvalue analysis. Fourth, in order to confirm the stability, the time responses for the system are computed from the nonlinear equations of motion. Finally, the experimental analysis proves theoretical stability and dynamic behavior of the PADB.

\section{Configuration of the PADB}

While the BADB has a circular disk with the race containing balls, which roll freely as shown in Fig. 1, the PADB has pivots, where the pendulums are suspended, around the center of the rotating system as shown in Fig. 2 . A schematic of the PADB is shown in Fig. 3.

The centroid $C$ of the rotating disk, which has angular velocity $\omega$, is defined by the polar coordinates $r$ and $\theta$. The center of mass $G$ is defined by eccentricity $\varepsilon$ and angle $\omega t-\theta$, which is the difference between axis $r$ and the line drawn from the centroid $C$ to the center of mass $G$. The pivot $D_{i}$, the rotating center of the $i$ th pendulum $P_{i}$, is defined by the distance $a$ from the centroid $C$ and angle $\varphi_{i}$ for $i=1,2, \cdots, n$ ( $n$ is the total number of the pendulums). And $P_{i}$ is defined by the pendulum distance $l$ and angle $\phi_{i}$ for $i=1,2, \cdots, n$. 


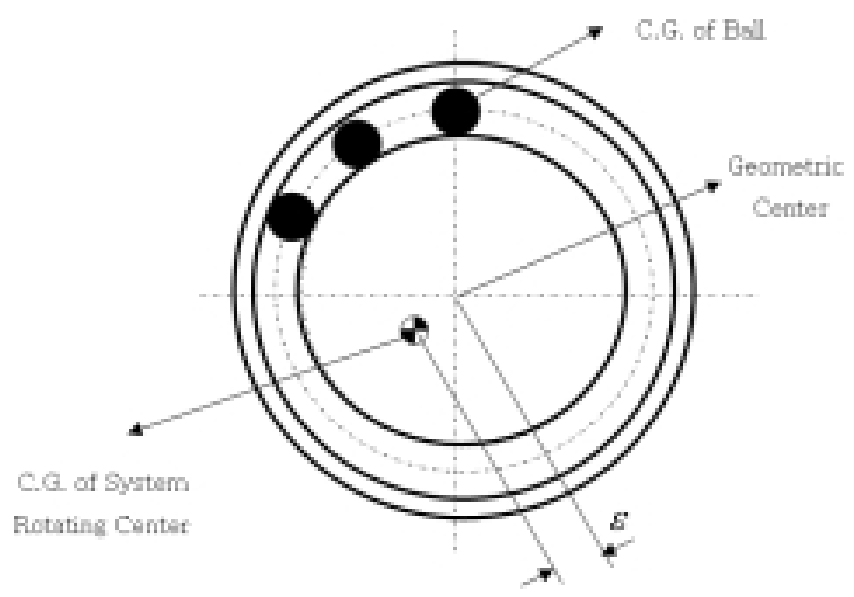

Fig. 1. Ball automatic dynamic balancer.

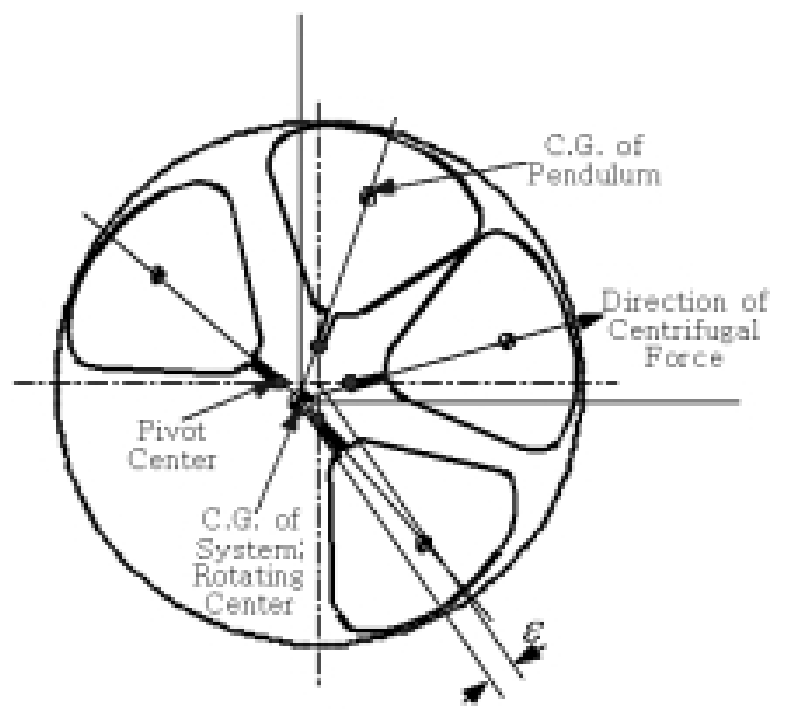

Fig. 2. Pendulum automatic dynamic balancer.

The nonlinear equations of motion of the system can be derived by the Lagrangian method, and the Lagrange's equation is given by

$$
\frac{d}{d t}\left(\frac{\partial T}{\partial \dot{q}_{k}}\right)-\frac{\partial T}{\partial q_{k}}+\frac{\partial V}{\partial q_{k}}=Q_{k}^{n c},
$$

where $T$ is the kinetic energy, $V$ is the potential energy, $Q_{k}^{n c}$ is the generalized non-conservative force and $q_{k}$ are the generalized coordinates. For PADB, the generalized coordinates are $r, \theta$ and $\phi_{1}, \phi_{2}, \ldots, \phi_{n}$, and $n+2$ independent equations of motion are derived by Lagrange's equation.

The position vector $\mathbf{r}_{\mathrm{G}}$ of the center of mass $G$ and the position vector $\mathbf{r}_{\mathrm{P}_{\mathrm{i}}}$ of the $i$ th pendulum $P_{i}$ are represented as

$$
\begin{aligned}
& \mathbf{r}_{\mathrm{G}}=[r+\varepsilon \cos (\omega t-\theta)] \mathbf{e}_{\mathrm{r}}+\varepsilon \sin (\omega t-\theta) \mathbf{e}_{\theta}, \\
& \mathbf{r}_{\mathrm{P}_{\mathrm{i}}}=\left[r+a \cos \left(\varphi_{i}+\omega t-\theta\right)+l \cos \left(\phi_{i}+\varphi_{i}+\omega t-\theta\right)\right] \mathbf{e}_{\mathrm{r}} \\
& \quad+\left[a \sin \left(\varphi_{i}+\omega t-\theta\right)+l \sin \left(\phi_{i}+\varphi_{i}+\omega t-\theta\right)\right] \mathbf{e}_{\theta}, \quad i=1,2, \cdots, n,
\end{aligned}
$$




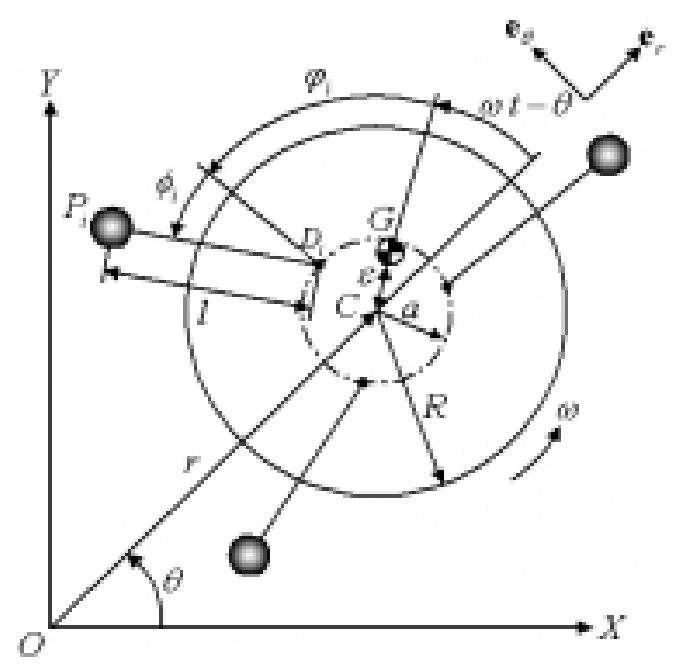

Fig. 3. Schematic of a pendulum automatic dynamic balancer.

where $\mathbf{e}_{\mathrm{r}}$ and $\mathbf{e}_{\theta}$ are the unit vectors in the $r$ and $\theta$ directions respectively. When $a$ and $\varphi_{i}$ are ignored, i.e. $a=0$, $\varphi_{i}=0$ in Eq. (3), the position vector of $i$ th ball of the BADB is obtained and that is same as the analysis of Chung [4].

Assuming that the pendulums have the same mass, the kinetic energy is expressed as

$$
T=\frac{1}{2} I_{G} \omega^{2}+\frac{1}{2} M \dot{\mathbf{r}}_{\mathrm{G}} \cdot \dot{\mathbf{r}}_{\mathrm{G}}+\frac{1}{2} m \sum_{i=1}^{n} \dot{\mathbf{r}}_{\mathrm{P}_{\mathrm{i}}} \cdot \dot{\mathbf{r}}_{\mathrm{P}_{\mathrm{i}}},
$$

where $I_{G}$ is the mass moment of inertia of the disk with respect to $G, M$ and $m$ are the mass of the disk and each pendulum, respectively. When the effect of gravity is ignored, the potential energy is expressed as

$$
V=\frac{1}{2} k r^{2},
$$

where $k$ is the equivalent stiffness of the system correspond to the stiffness of the shaft carrying the disk. The generalized non-conservative force is expressed as

$$
\begin{aligned}
Q_{r}^{n c} & =-c \dot{r}, \\
Q_{\theta}^{n c} & =c r^{2} \dot{\theta}, \\
Q_{\phi_{i}}^{n c} & =-D \dot{\phi}_{i}, \quad i=1,2, \cdots, n,
\end{aligned}
$$

where $c$ is the equivalent viscous damping coefficient of the shaft carrying the disk and $D$ is the viscous drag coefficient at the pivot of pendulum.

The nonlinear equations of motion are obtained by substituting Eqs (4)-(8) into Eq. (1) and given by

$$
\begin{aligned}
& (M+n m)\left(\ddot{r}-r \dot{\theta}^{2}\right)+k r+c \dot{r}-m \sum_{i=1}^{n}\left[l \ddot{\phi}_{i} \sin \left(\phi_{i}+\varphi_{i}+\omega t-\theta\right)\right. \\
& \left.+l\left(\dot{\phi}_{i}+\omega\right)^{2} \cos \left(\phi_{i}+\varphi_{i}+\omega t-\theta\right)+a \omega^{2} \cos \left(\varphi_{i}+\omega t-\theta\right)\right]=M \varepsilon \omega^{2} \cos (\omega t-\theta), \\
& (M+n m)(r \ddot{\theta}+2 \dot{r} \dot{\theta})+c r \dot{\theta}+m \sum_{i=1}^{n}\left[l \ddot{\phi}_{i} \cos \left(\phi_{i}+\varphi_{i}+\omega t-\theta\right)\right. \\
& \left.-l\left(\dot{\phi}_{i}+\omega\right)^{2} \sin \left(\phi_{i}+\varphi_{i}+\omega t-\theta\right)-a \omega^{2} \sin \left(\varphi_{i}+\omega t-\theta\right)\right]=M \varepsilon \omega^{2} \sin (\omega t-\theta),
\end{aligned}
$$




$$
\begin{aligned}
& m l^{2} \ddot{\phi}_{i}+D \dot{\phi}_{i}-m l\left(\ddot{r}-r \dot{\theta}^{2}\right) \sin \left(\phi_{i}+\varphi_{i}+\omega t-\theta\right) \\
& +m l(2 \dot{r} \dot{\theta}+r \ddot{\theta}) \cos \left(\phi_{i}+\varphi_{i}+\omega t-\theta\right)+m a l \omega^{2} \sin \phi_{i}=0, \quad i=1,2, \cdots, n .
\end{aligned}
$$

Since $\theta$ increases monotonically with respect to time, it is impossible to compute the equilibrium position with respect to $\theta$. Therefore, a generalized coordinate $\psi$ that represents the angle from the $r$ direction to the center of mass $G$ is introduced as

$$
\psi=\omega t-\theta \text {. }
$$

In order to analyze the stability of the system conveniently, the state equations are introduced by arranging equations (9)-(11) and written by a matrix form

$$
\begin{aligned}
& \mathbf{A}(\mathbf{x}) \dot{\mathbf{x}}=\mathbf{B}(\mathbf{x}), \\
& \mathbf{x}=\left[r \psi \phi_{1} \phi_{2} \cdots \phi_{n} \dot{r} \dot{\psi} \dot{\phi}_{1} \dot{\phi}_{2} \cdots \dot{\phi}_{n}\right]^{T} \text {, } \\
& \mathbf{A}=\left[\begin{array}{ll}
\mathbf{I} & \mathbf{O} \\
\mathbf{O} & \mathbf{M}
\end{array}\right]
\end{aligned}
$$

where $\mathbf{I}$ is $(n+2) \times(n+2)$ identity matrix, $\mathbf{M}$ is $(n+2) \times(n+2)$ mass matrix which is the function of displacement, and $\mathbf{B}$ is $(n+2) \times 1$ internal force matrix which is function of displacement and velocity.

\section{Equilibrium position and linearized equation}

Before the equilibrium positions are computed, the equilibrium position vector $\mathbf{x}^{*}$ is defined by

$$
\mathbf{x}^{*}=\left[r^{*} \psi^{*} \phi_{1}^{*} \phi_{2}^{*} \cdots \phi_{n}^{*} \dot{r}^{*} \dot{\psi}^{*} \dot{\phi}_{1}^{*} \dot{\phi}_{2}^{*} \cdots \dot{\phi}_{n}^{*}\right]^{T}
$$

where the symbol of asterisk means the value at the equilibrium position.

As at the equilibrium state there is no motion, by letting $\dot{\mathrm{x}}^{*}=\mathbf{0}$ in equation (13), the equilibrium positions can be computed from

$$
\mathbf{B}\left(\mathbf{x}^{*}\right)=\mathbf{0} \text {. }
$$

The constant $r^{*}, \psi^{*}, \phi_{i}^{*}, \dot{r}^{*}, \dot{\psi}^{*}$ and $\dot{\phi}_{i}^{*}$ are determined by solving the above $2 n+4$ algebraic equations, and the algebraic equations can be simplified as

$$
\begin{aligned}
& \dot{r}^{*}=0, \\
& \dot{\psi}^{*}=0, \\
& \dot{\phi}_{i}^{*}=0, \quad i=1,2, \cdots, n, \\
& {\left[k-(M+n m) \omega^{2}\right] r^{*}-m \omega^{2} \sum_{i=1}^{n}\left[l \cos \left(\phi_{i}^{*}+\varphi_{i}+\psi^{*}\right)+a \cos \left(\varphi_{i}+\psi^{*}\right)\right]==M \varepsilon \omega^{2} \cos \psi^{*},} \\
& c \omega r^{*}-m \omega^{2} \sum_{i=1}^{n}\left[l \sin \left(\phi_{i}^{*}+\varphi_{i}+\psi^{*}\right)+a \sin \left(\varphi_{i}+\psi^{*}\right)\right]=M \varepsilon \omega^{2} \sin \psi^{*}, \\
& r^{*} \sin \left(\phi_{i}^{*}+\varphi_{i}+\psi^{*}\right)+a \sin \phi_{i}^{*}=0, \quad i=1,2, \cdots, n .
\end{aligned}
$$

The BADB has two equilibrium positions, the balanced and unbalanced case, which correspond to $r^{*}=0$ and $r^{*} \neq 0$, respectively. In the balanced case of the BADB, i.e. $r^{*}=0$, the amount of total unbalance of system can be reduced to zero. Principally, in contrast to the BADB, PADB can not completely compensate the unbalance of 
rotating system. It means that in case of PADB $r^{*}$ in the balanced case is not zero. The reason is that if $r^{*}$ goes zero the pendulums go to the positions where $\phi_{i}^{*}=0$ and this induces unbalance of rotor again. Since $r^{*}$ is not zero in the balanced case of the PADB, $r^{*}$ can be eliminated in the Eqs (21)-(23):

$$
\begin{aligned}
& {\left[c M \varepsilon \omega+c m \omega \sum_{i=1}^{n}\left\{l \cos \left(\phi_{i}^{*}+\varphi_{i}\right)+a \cos \varphi_{i}\right\}\right.} \\
& \left.-m\left\{k-(M+n m) \omega^{2}\right\} \sum_{i=1}^{n}\left[l \sin \left(\phi_{i}^{*}+\varphi_{i}\right)+a \sin \varphi_{i}\right]\right] \cos \psi^{*} \\
& -\left[M \varepsilon\left\{k-(M+n m) \omega^{2}\right\}+c m \omega \sum_{i=1}^{n}\left\{l \sin \left(\phi_{i}^{*}+\varphi_{i}\right)+a \sin \varphi_{i}\right\}\right. \\
& \left.+m\left\{k-(M+n m) \omega^{2}\right\} \sum_{i=1}^{n}\left\{l \cos \left(\phi_{i}^{*}+\varphi_{i}\right)+a \cos \varphi_{i}\right\}\right] \sin \psi^{*}=0 .
\end{aligned}
$$

Two non-trivial solutions in Eq. (24) are obtained as

$$
\begin{aligned}
& c M \varepsilon \omega+c m \omega \sum_{i=1}^{n}\left[l \cos \left(\phi_{i}^{*}+\varphi_{i}\right)+a \cos \varphi_{i}\right] \\
& -m\left[k-(M+n m) \omega^{2}\right] \sum_{i=1}^{n}\left[l \sin \left(\phi_{i}^{*}+\varphi_{i}\right)+a \sin \varphi_{i}\right]=0, \\
& M \varepsilon\left[k-(M+n m) \omega^{2}\right]+c m \omega \sum_{i=1}^{n}\left[l \sin \left(\phi_{i}^{*}+\varphi_{i}\right)+a \sin \varphi_{i}\right] \\
& +m\left[k-(M+n m) \omega^{2}\right] \sum_{i=1}^{n}\left[l \cos \left(\phi_{i}^{*}+\varphi_{i}\right)+a \cos \varphi_{i}\right]=0 .
\end{aligned}
$$

If it is assumed that the PADB has two pendulums $(n=2)$ and $\varphi_{1}$ and $\varphi_{2}$ are $\frac{\pi}{2}$ and $\frac{3 \pi}{2}$, respectively, the equilibrium positions for the pendulums are obtained as follows:

$$
\begin{aligned}
& \sin \phi_{1}^{*}=\frac{M \varepsilon}{2 m l}, \\
& \sin \phi_{2}^{*}=-\frac{M \varepsilon}{2 m l}, \\
& \cos \phi_{1}^{*}=\cos \phi_{2}^{*}=\frac{\sqrt{4 m^{2} l^{2}-M^{2} \varepsilon^{2}}}{2 m l} .
\end{aligned}
$$

In order to find the similarity between the balanced cases of the PADB and the BADB, computation results are investigated when $r^{*}=0$ in the Eqs (21)-(23) as follows:

$$
\begin{aligned}
& \frac{m}{M} \sum_{i=1}^{n}\left[\cos \left(\phi_{i}^{*}+\varphi_{i}\right)+\frac{a}{l} \cos \varphi_{i}\right]+\frac{\varepsilon}{l}=0, \\
& \sum_{i=1}^{n}\left[\sin \left(\phi_{i}^{*}+\varphi_{i}\right)+\frac{a}{l} \sin \varphi_{i}\right]=0 .
\end{aligned}
$$


Under the simplified condition, i.e. $n=2, \varphi_{1}=\frac{\pi}{2}$ and $\varphi_{2}=\frac{3 \pi}{2}$, in Eqs (30) and (31), the same results found in Eqs (27)-(29) are obtained. Therefore, the dynamic behavior of pendulums in the balanced case of the PADB corresponds to the balanced case of the BADB even if $r^{*}$ is not zero in the balanced case of the PADB. Since the equilibrium positions in the unbalanced case of the PADB, i.e. $r^{*} \neq 0$, are too complicated to express them in closed form, and it is important that rotating system should be balanced, this paper will focus on the balanced case.

In order to obtain the linearized equations around equilibrium position, the perturbation method is applied to the nonlinear equation of motion as defined in Eq. (13). To apply the perturbation method, solutions of Eq. (13) are assumed in the form of a power series in the small quantity $\delta$

$$
\begin{aligned}
& r=r^{*}+\delta r_{1}+\delta^{2} r_{2}+\cdots, \\
& \psi=\psi^{*}+\delta \psi_{1}+\delta^{2} \psi_{2}+\cdots, \\
& \phi_{i}=\phi_{i}^{*}+\delta \phi_{i_{1}}+\delta^{2} \phi_{i_{2}}+\cdots, \quad i=1,2, \cdots, n,
\end{aligned}
$$

where $r^{*}, \psi^{*}$ and $\phi_{i}^{*}$ represent the equilibrium positions for $r, \psi$ and $\phi_{i}$, respectively. Substituting Eqs (32)-(34) to Eq. (13) and collecting all terms with respect to $\delta$, equilibrium positions and linearized equations around equilibrium positions are obtained.

The linearized equations around the equilibrium positions can be expressed by matrix form

$$
\mathbf{A}^{*} \dot{\mathbf{x}}_{\mathrm{p}}=\mathbf{B}^{*} \mathbf{x}_{\mathrm{p}}
$$

where $x_{p}$ is the perturbation of $\mathbf{x}$,

$$
\mathbf{x}_{\mathrm{p}}=\left[r_{1} \psi_{1} \phi_{1_{1}} \phi_{2_{1}} \cdots \phi_{n_{1}} \dot{r}_{1} \dot{\psi}_{1} \dot{\phi}_{1_{1}} \dot{\phi}_{2_{1}} \cdots \dot{\phi}_{n_{1}}\right]^{T} .
$$

$\mathbf{A}^{*}$ and $\mathbf{B}^{*}$ are defined by

$$
\begin{aligned}
& \mathbf{A}^{*}=\mathbf{A}\left(\mathbf{x}^{*}\right), \\
& \mathbf{B}^{*}=\left[\begin{array}{ll}
\mathbf{0} & \mathbf{I} \\
\mathbf{K}^{*} & \mathbf{C}^{*}
\end{array}\right],
\end{aligned}
$$

where $\mathbf{I}$ is $(n+2) \times(n+2)$ identity matrix, and are $(n+2) \times(n+2)$ constant matrices.

\section{Stability analysis}

The stability around the equilibrium positions is determined by linearized equation given by Eq. (35). In order to simplify the investigation of the stability, it is assumed that the PADB have two pendulums, i.e. $n=2$. Then $\mathbf{A}^{*}$ and $\mathbf{B}^{*}$ become $8 \times 8$ matrices. The stability can be investigated by solving eigenvalue problem for Eq. (35). A solution of Eq. (35) would be

$$
\mathbf{x}_{\mathrm{p}}=\mathbf{X}_{\mathrm{p}} e^{\lambda t},
$$

where $\lambda$ is an eigenvalue and $\mathbf{X}_{\mathrm{p}}$ is an eigenvector corresponding to $\lambda$. By substituting Eqs (39) to (35), the eigenvalue problem is defined

$$
\left(\mathbf{B}^{*}-\lambda \mathbf{A}^{*}\right) \mathbf{X}_{\mathrm{p}}=0 .
$$

When all the eigenvalues have negative real parts, the system is stable. However, if only one of the eigenvalues has a positive real part, the system becomes unstable. The eigenvalues can be determined by solving the following characteristic equation

$$
\operatorname{det}\left(\mathbf{B}^{*}-\lambda \mathbf{A}^{*}\right)=0
$$

which can be expressed as a polynomial of $\lambda$ : 


$$
\begin{aligned}
& c_{0}\left(\frac{\lambda}{\omega_{n}}\right)^{8}+c_{1}\left(\frac{\lambda}{\omega_{n}}\right)^{7}+c_{2}\left(\frac{\lambda}{\omega_{n}}\right)^{6}+c_{3}\left(\frac{\lambda}{\omega_{n}}\right)^{5}+c_{4}\left(\frac{\lambda}{\omega_{n}}\right)^{4}+c_{5}\left(\frac{\lambda}{\omega_{n}}\right)^{3}+c_{6}\left(\frac{\lambda}{\omega_{n}}\right)^{2} \\
& +c_{7} \frac{\lambda}{\omega_{n}}+c_{8}=0
\end{aligned}
$$

where $c_{i}$ are constants and $\omega_{n}$ is the natural frequency of the system defined by

$$
\omega_{n}=\sqrt{k / M} .
$$

Since Eq. (40) represents eight linear algebraic equations with nine unknowns, the equilibrium position of $\psi^{*}$ is not defined in the case of balanced case, therefore, the characteristic equation cannot be computed from Eq. (40) and an additional equation is needed. This equation is given by

$$
\sin ^{2} \psi^{*}+\cos ^{2} \psi^{*}=1 .
$$

Eliminating $\psi^{*}$ from Eqs (40) and (44), the characteristic equation is obtained in the form of Eq. (42). And the characteristic equation is simplified by using non-dimensional parameters defined by

$$
\begin{aligned}
& \tilde{r}=\frac{r}{l}, \quad \tilde{m}=\frac{m}{M}, \quad \tilde{\varepsilon}=\frac{\varepsilon}{l}, \quad \tilde{a}=\frac{a}{l}, \quad \tilde{\omega}=\frac{\omega}{\omega_{n}}, \\
& \beta=\frac{D}{m l \omega_{n}^{2}}, \quad \tilde{t}=\omega_{n} t, \quad \zeta=\frac{c}{2 \sqrt{M k}} .
\end{aligned}
$$

Physically, an unstable system, whose natural response grows without bound, can cause damage to the system. The stability or instability of the system depends on the roots of the polynomial Eq. (42). Since finding the roots of the polynomial Eq. (42) takes long time, a simplified procedure, known as the Routh-Hurwitz stability criterion, can be used to investigate the stability of the system.

All the coefficients of the Eq. (42) contain only non-dimensional parameters, hence the stability of the system is investigated for the variation of the non-dimensional parameters. Since it is not efficient to investigate the stability considering the variations of all the system parameters, the stability is checked with the variations for selected pairs of principal parameters such as rotating speed of the system, mass of pendulum, damping coefficient of the system and eccentricity. For comparison of the PADB and the BADB, selected pairs of parameters are same as those of stability analysis conducted by Chung [4].

In Figs 4-8, the results of stability analysis of the PADB and the BADB are compared. As mentioned above, stability of the BADB is investigated from the Eq. (41) in which $a$ and $\varphi_{i}$ are ignored, i.e. $a=0, \varphi_{i}=0$. Each graph (a) of Figs 4-8 represents the stability of the PADB, while each graph (b) represents the stability of the BADB. In the graphs, the dotted region means the stable region for the balanced equilibrium positions of the ADB, while the non-dotted region means the unstable region. If the system parameters are inside the stable region in each graph, the ADB is working and the system becomes balanced. On the other hand, the ADB cannot reduce vibration of the system in the non-dotted region. The stability of the ADB is investigated for $\tilde{m}=0.117, \tilde{a}=0.124, \tilde{\varepsilon}=0.0017$, $\tilde{\omega}=2.5, \zeta=0.106$ and $\beta=3.095$.

Figure 4 shows that the influence of $\tilde{m}$ and $\tilde{\varepsilon}$ on the stability of the system. The graphs show that the PADB has broader stable region than that of the BADB and the graph of the BADB shows that the system is not stable for $\tilde{m}<\tilde{\varepsilon} / 2$. Therefore, if each system is working with a fixed value of eccentricity and other parameters are same, the PADB has larger allowable eccentricity than that of the BADB. Figure 5 shows the influence of the energy dissipation factor $\beta$ on the stability of the system. Notice that the system with the PADB cannot be balanced when $\tilde{\omega}$ is near 2 and $\beta$ is small. While $\beta$ is relevant to damping fluid in the groove containing balls in case of the BADB, it is relevant to damping coefficient of bearing of pivot around the center of rotating system in case of the PADB.

Figures 6-8 show the influence of the eccentricity of the rotor, the mass of the pendulum or ball and another energy dissipation factor $\varsigma$ on the stability of the system. That is, the stability for the variations of $\beta, \tilde{\varepsilon}, \tilde{m}, \varsigma$ and $\tilde{\varepsilon}$ versus $\tilde{\omega}$ is presented in Figs 5-8. The graphs show that the system with the BADB cannot be balanced for $\tilde{\omega}<1$. However, with the exception of $\tilde{\omega}$ near 1, the system with the PADB can be balanced even if $\tilde{\omega}<1$. That is, if each system is working with same parameters, the PADB is more stable than the BADB for the variation of $\tilde{\omega}$.

Figures 4-8 provide the design criteria of the ADB for the design parameters in order for the system to be stable. That is, design parameters of the ADB are required to have the value of stable region and then the vibration of the system can be reduced. 


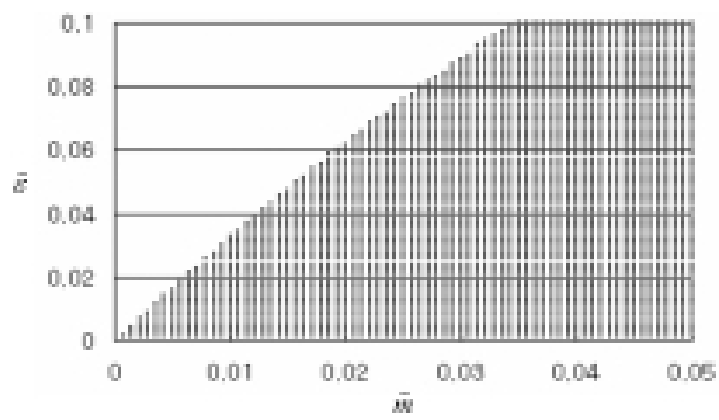

(a)

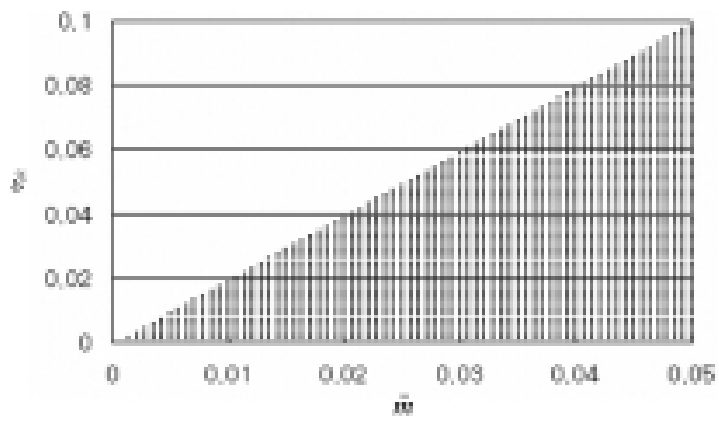

(b)

Fig. 4. Comparison of stability for the variation of $\tilde{m}$ and $\tilde{\varepsilon}$. (a) Pendulum Automatic Dynamic Balancer. (b) Ball Automatic Dynamic Balancer.

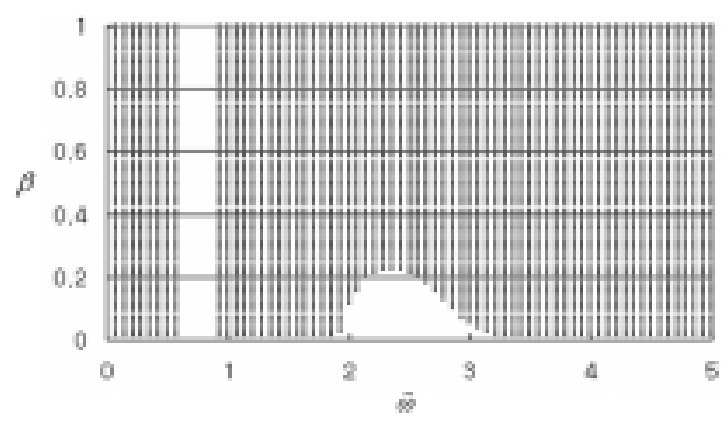

(a)

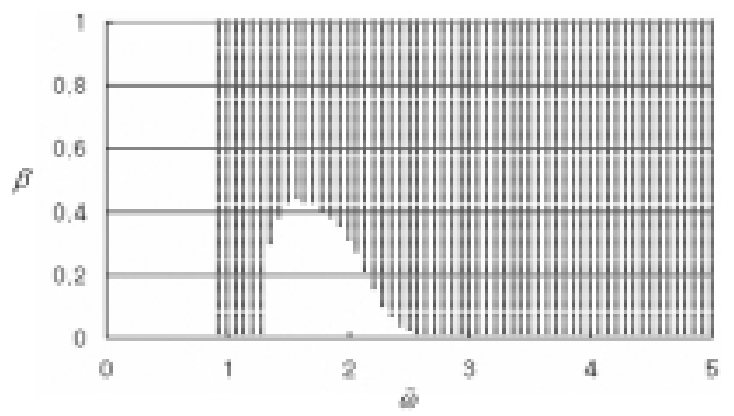

(b)

Fig. 5. Comparison of stability for the variation of $\tilde{\omega}$ and $\beta$. (a) Pendulum Automatic Dynamic Balancer. (b) Ball Automatic Dynamic Balancer. 


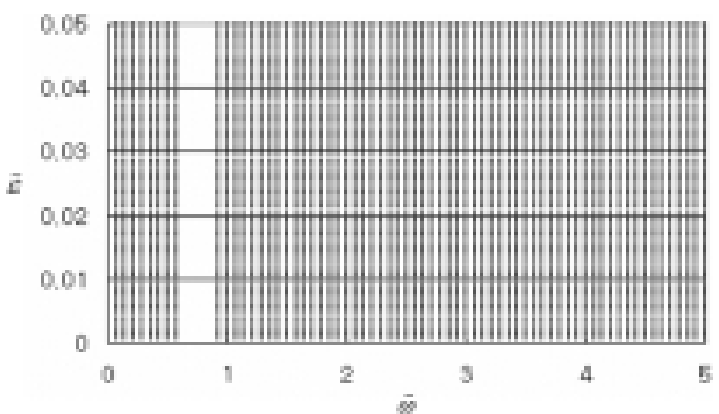

(a)

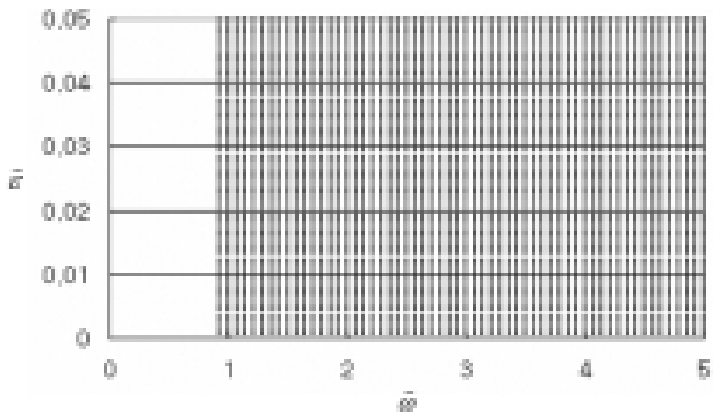

(b)

Fig. 6. Comparison of stability for the variation of $\tilde{\omega}$ and $\tilde{\varepsilon}$. (a) Pendulum Automatic Dynamic Balancer. (b) Ball Automatic Dynamic Balancer.

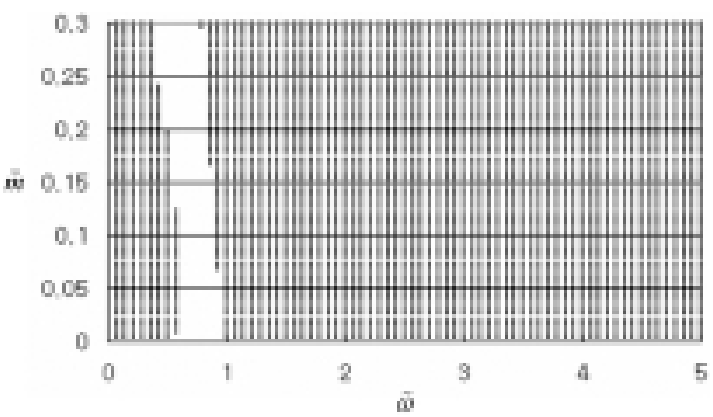

(a)

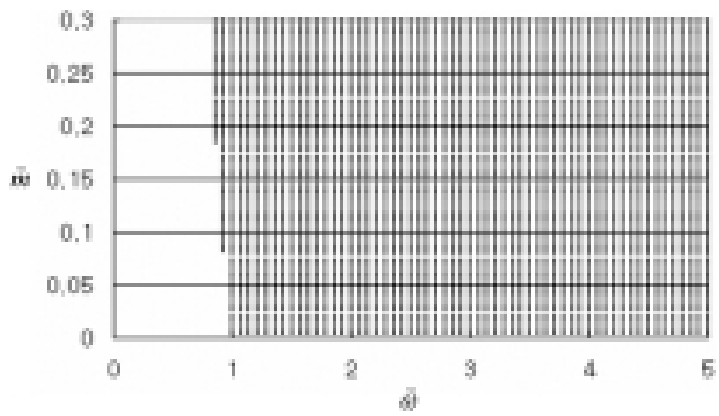

(b)

Fig. 7. Comparison of stability for the variation of $\tilde{\omega}$ and $\tilde{m}$. (a) Pendulum Automatic Dynamic Balancer. (b) Ball Automatic Dynamic Balancer. 


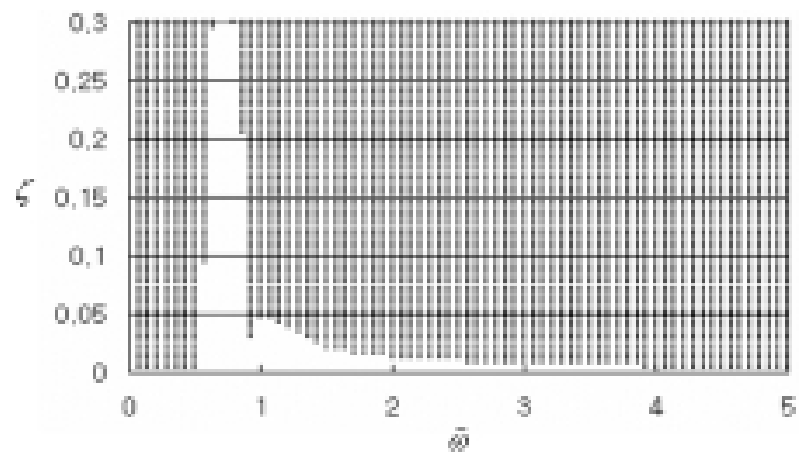

(a)

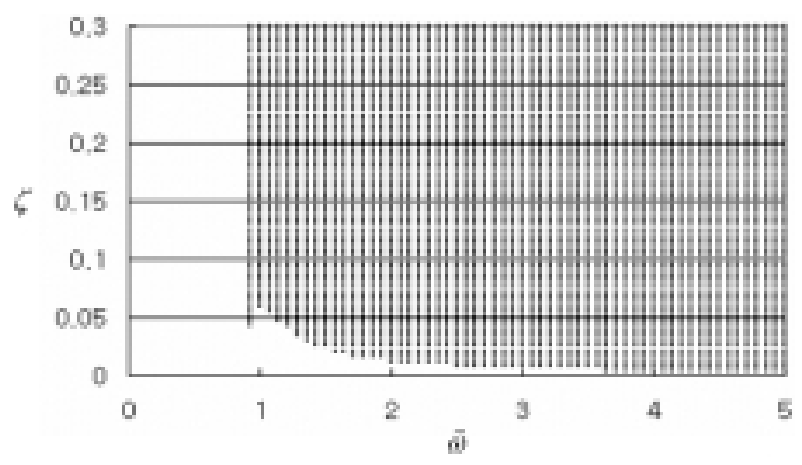

(b)

Fig. 8. Comparison of stability for the variation of $\tilde{\omega}$ and $\varsigma$. (a) Pendulum Automatic Dynamic Balancer. (b) Ball Automatic Dynamic Balancer.

\section{Simulation results of time responses}

Direct time integration of the equations of motion by computer simulation provides an evidence for the stability investigation of a general time varying motion. Time responses are computed for the stable and unstable region. The physical system parameters for computation are given by $M=0.0797 \mathrm{~kg}, m=0.0093 \mathrm{~kg}, k=1116 \mathrm{~N} / \mathrm{m}$, $l=0.0242 \mathrm{~m}, a=0.003 \mathrm{~m}, \varepsilon=7.528 \times 10^{-5} \mathrm{~m}, c=2 \mathrm{Ns} / \mathrm{m}$, and $D=2 \times 10^{-3} \mathrm{Nm} / \mathrm{s}$. Thus the corresponding non-dimensional parameters are $\tilde{m}=0.116, \tilde{a}=0.124, \tilde{\varepsilon}=0.003, \varsigma=0.106$, and $\beta=3.103$. In addition, the natural frequency of the system is $\omega_{n}=118.332 \mathrm{rad} / \mathrm{s}$. The initial conditions are given by $r(0)=1 \times 10^{-3} \mathrm{~m}$, $\psi(0)=0, \phi_{1}(0)=45^{\circ}, \phi_{2}(0)=90^{\circ}$ and $\dot{r}(0)=\dot{\psi}(0)=\dot{\phi}_{1}(0)=\dot{\phi}_{2}(0)=0$.

The time responses of the positions of the pendulums and balls are shown in Figs 9-11, in which the behavior of the PADB is compared to that of the BADB. Figure 9 shows the time responses of the systems, with the pendulum and ball ADBs, are computed for $\tilde{\omega}=2.5$ and $\tilde{m}=0.116$, which correspond to the stable region of in Fig. 7 . The radial displacement of ball converges to zero and that of pendulum converges to a small constant value over time. In addition, the radial displacement of system without any balancer converges to constant value larger than the value of pendulum's convergence. It means that the radial displacement and pendulum positions approach the equilibrium position and the vibration of the system is reduced.

Similarly, Fig. 10 shows the time response of the systems with the pendulum and ball ADBs for $\tilde{\omega}=0.9$ and $\tilde{m}=0.116$, which correspond to the unstable region in Fig. 7. The radial displacement of system with the pendulum or ball ADB diverges, while radial displacement of system without any balancer converges to constant value.

In addition, Fig. 11 shows the time response of the systems with the pendulum and ball ADBs for $\tilde{\omega}=0.2$ and $\tilde{m}=0.116$, which correspond to the stable region for only PADB in Fig. 7. Since the radial displacement of the system with the PADB converges to a small constant value, the PADB is working for $\tilde{\omega}=0.2$. However, the radial displacement of the system with the BADB is not so small as to reduce vibration of the system. As a result of 


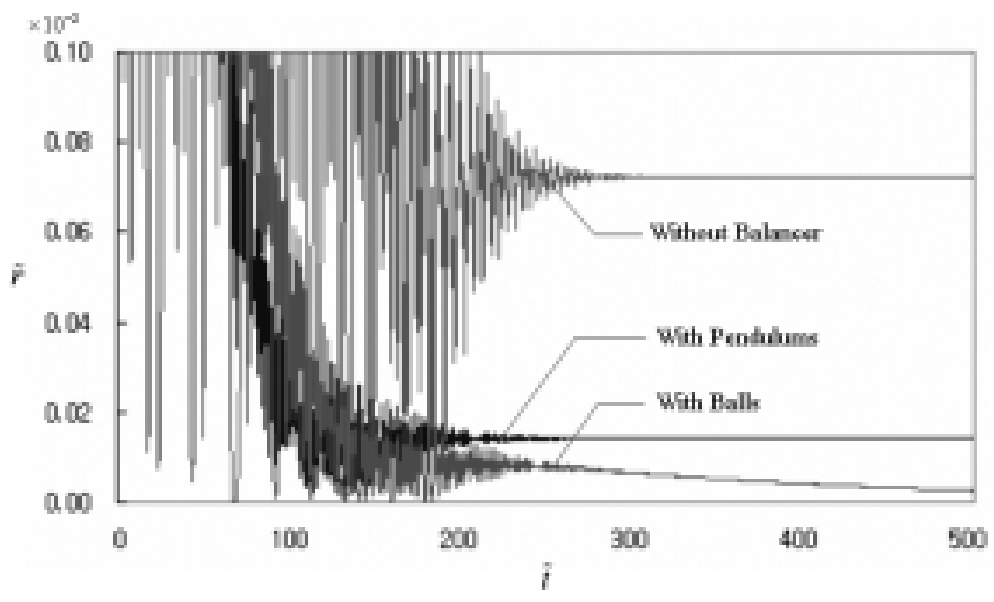

(a)

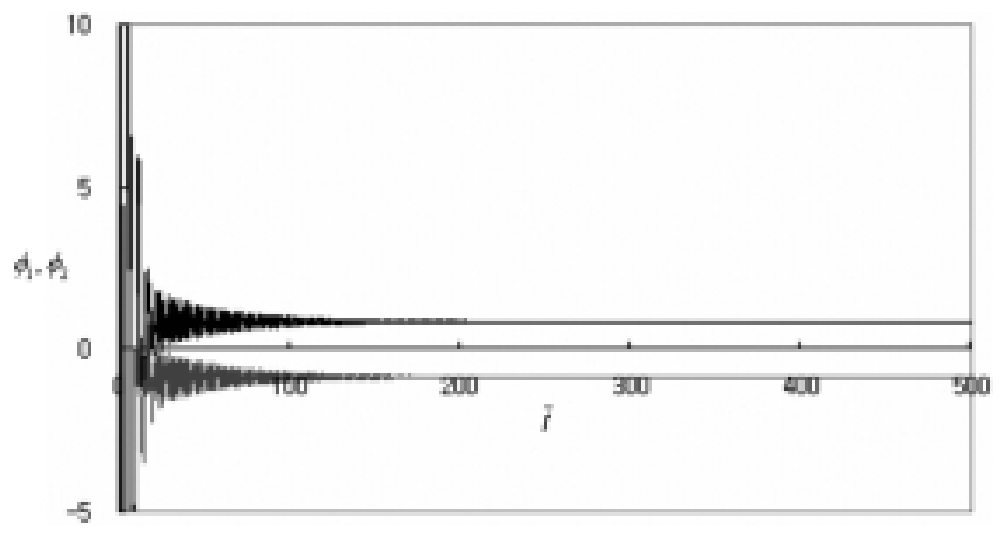

(b)

Fig. 9. Time response of system with Pendulum or Ball Automatic Dynamic Balancer for $\tilde{\omega}=2.5$ and $\tilde{m}=0.116$. (a) Time response of the radial displacement. (b) Time response of the pendulum position.

comparisons of the PADB with the BADB, the PADB is more helpful for the system in obtaining balancing than the BADB for $\tilde{\omega}<0.6$.

\section{Experimental result}

The dynamic stability and time responses were analyzed in the previous chapter. In order to prove the analytical results, various experiments are made on the PADB with the experimental setup shown in Fig. 12. The rotating system is supported by rubber suspensions at 3 points and the suspending natural frequency in the direction of unbalanced motion is set to $25 \mathrm{~Hz}$. For compatibility with analytical results, rotating disk and a couple of pendulums having same weight are made and ball bearings are used for pendulums to hang on rotating disk. Pendulum Motion limit stops confining movement of pendulums are installed near the pendulums. Without pendulum motion limit stops, it is difficult for PADB to reach the steady state because excessive motion of pendulums instantaneously increases the unbalance of rotating system, and that hinders the rotating system from passing over the suspension natural frequency. When the pendulum motion limit stops are so close to the pendulums, the pendulums can not 


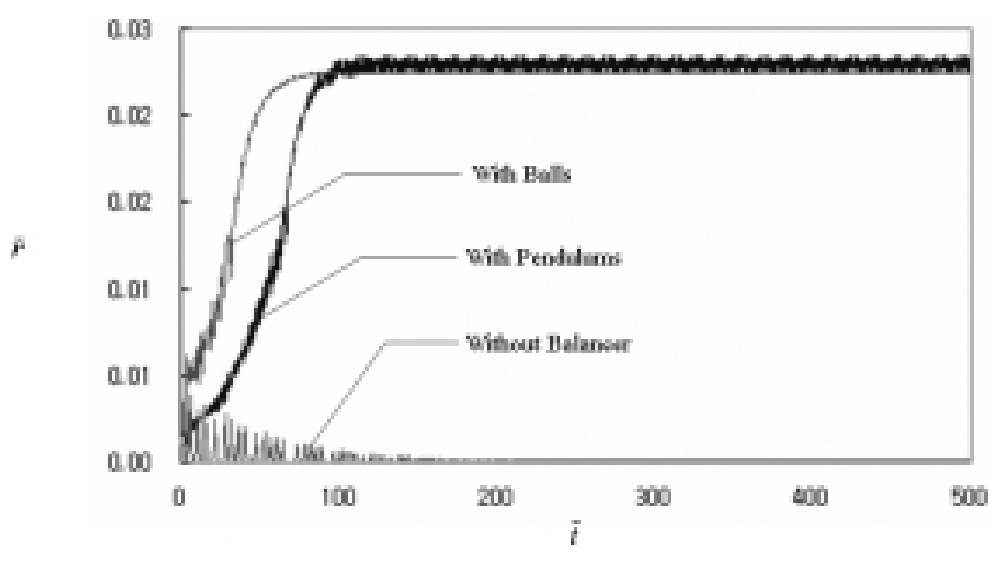

(a)

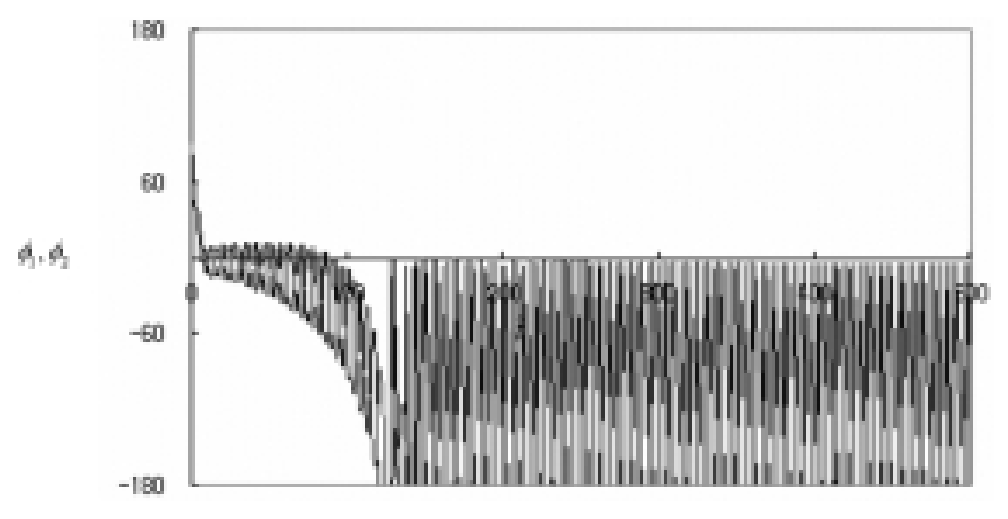

(b)

Fig. 10. Time response of system with Pendulum or Ball Automatic Dynamic Balancer for $\tilde{\omega}=0.9$ and $\tilde{m}=0.116$. (a) Time response of the radial displacement. (b) Time response of the pendulum position.

move to the appropriate positions for maximum balancing. So, motion limit stops are installed as much angle as 10 degrees from the edge of the pendulums on the basis of simulation result that steady state rotated angle of pendulums are within 5 degrees in this experimental setup. With small size unbalancing mass attached on rotating disk, the radial displacement of unbalanced rotating system is measured by accelerometer.

In order to evaluate the ability of the PADB, the vibration reduction ratio $\eta$ is defined in Eq. (46). In other words, it is the ratio of the decreased amount of unbalance to original amount of unbalance

$$
\eta=\frac{\varepsilon-r}{\varepsilon} \times 100
$$

The experimental results for $\tilde{m}=0.116, \tilde{a}=0.124$, and $\tilde{\varepsilon}=0.003$ are shown in Fig. 13. A negative sign of vibration reduction ratio means that the PADB cannot reduce vibration of the system any more. In other words, the pendulums increase the total amount of unbalance for the system.

For $\tilde{\omega}$ of 0.3 to 1.1 , the experimental vibration reduction is different from the theoretical vibration reduction as shown in Fig. 13. However, it is believed that the experiment is influenced by minor differences of natural frequency of the experimental system and it is not easy to measure the radial displacement of the system due to the large vibration. For $\tilde{\omega}>1.3$ or $\tilde{\omega}<0.3$, the experimental results agree well with the theoretical results. 


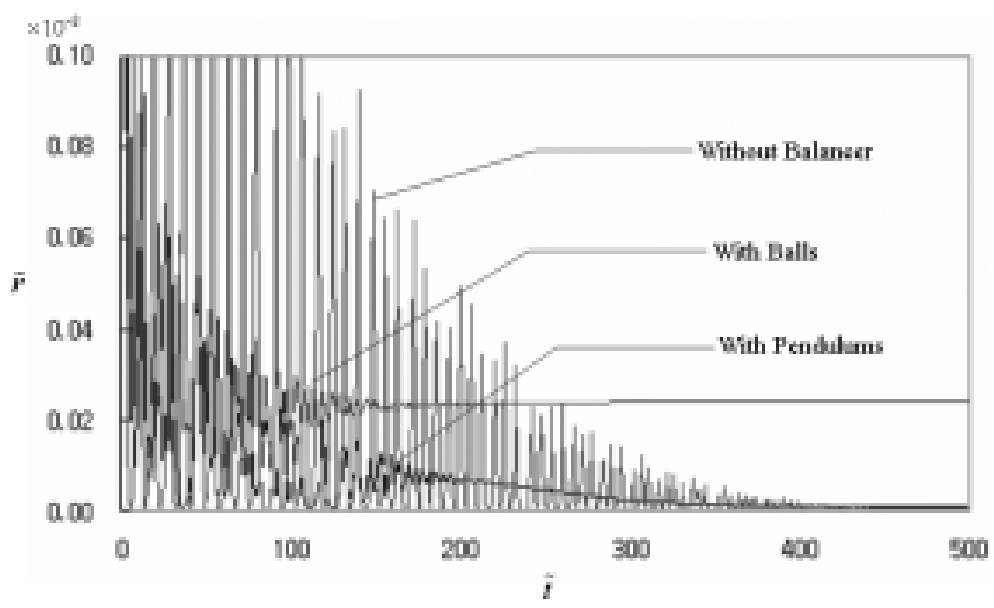

(a)

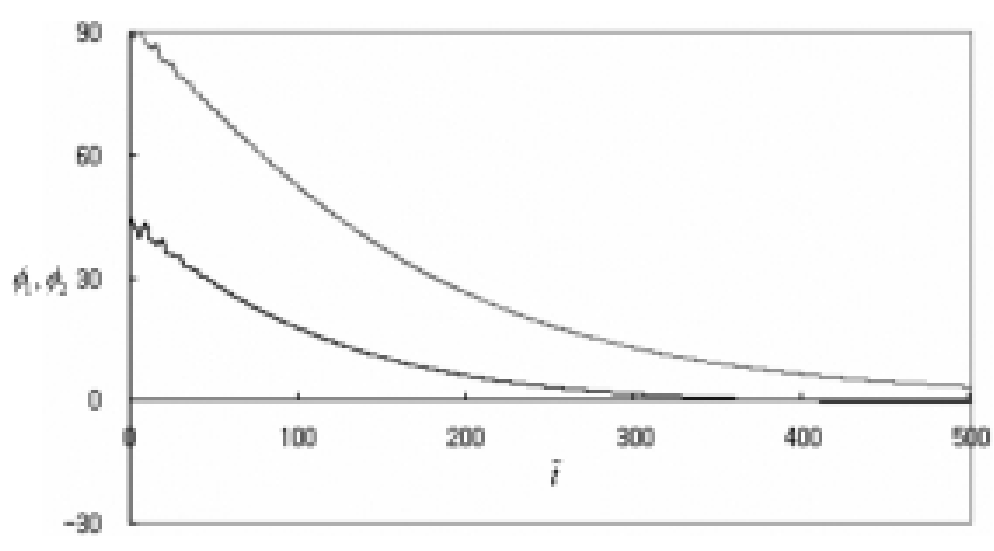

(b)

Fig. 11. Time response of system with Pendulum or Ball Automatic Dynamic Balancer for $\tilde{\omega}=0.2$ and $\tilde{m}=0.116$. (a) Time response of the radial displacement. (b) Time response of the pendulum position.

\section{Conclusions}

In this paper, the stability and time responses for the PADB are analyzed and compared with those of the BADB. The non-linear equations of motion for the PADB and their linear variational forms around equilibrium positions are driven. The Routh-Hurwitz criteria are applied to stability analysis for various parameters that have influences on the stability of the system with the PADB, such as rotating speed, mass of pendulum, damping coefficient of the pivot, and eccentricity. The stable region of each parameter can be used as a design constraint for the PADB. The analysis show that the vibration reduction efficiency of the BADB is better than that of the PADB, when the rotating speed is above the critical speed, i.e. $\tilde{\omega}>1$. However, the PADB has broader stable regions than the BADB. For example, the PADB can achieve balancing for $\tilde{\omega}<0.6$, where such a region is unstable for the BADB. Finally, experimental results agree with those of the simulation, therefore, it is confirmed experimentally that the PADB can be applied to rotating systems to achieve balancing. 


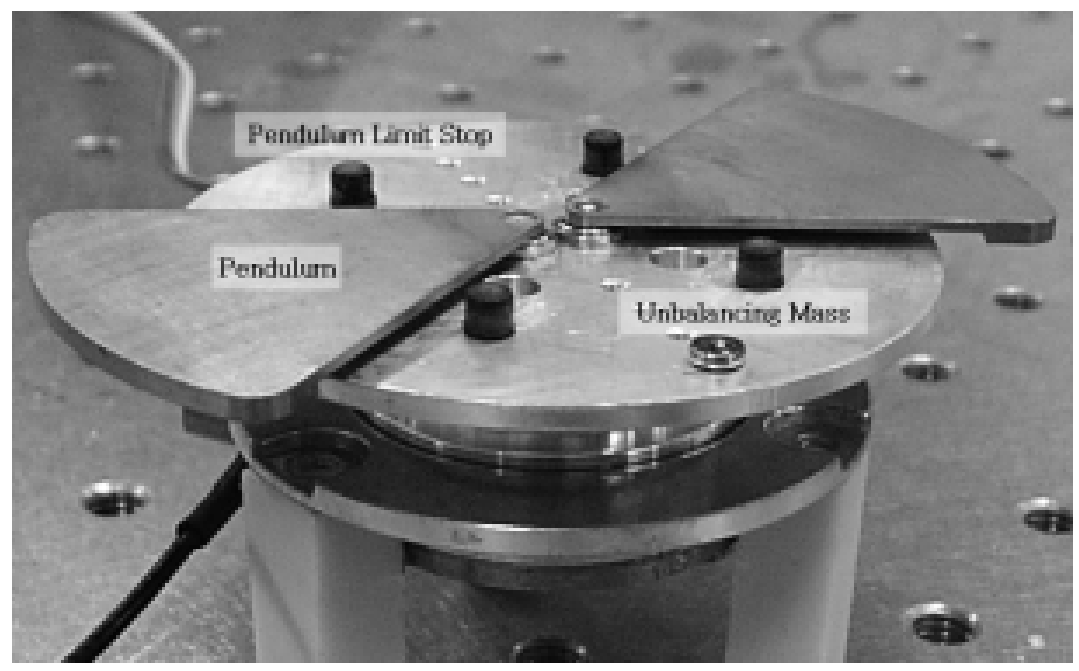

Fig. 12. Experimental setup.

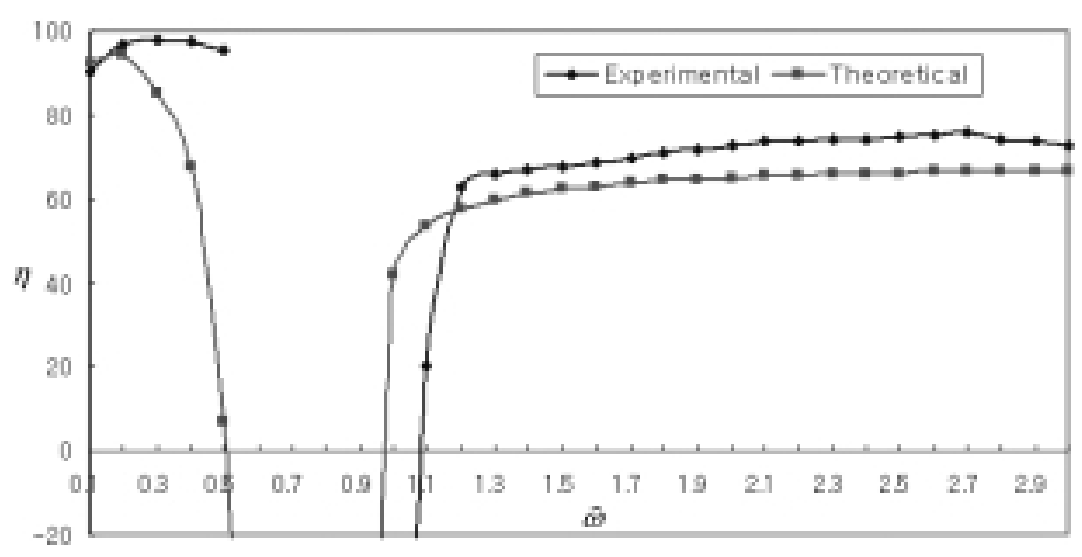

Fig. 13. Vibration reduction ratio.

\section{Acknowledgments}

This work was funded by the Korea Science and Engineering Foundation (KOSEF) through the Center for Information Storage Device (CISD) Grant No. R11-1997-042-11003-0.

\section{APPENDICES}

$$
\mathbf{M}=\left[\begin{array}{lllll}
M+n m & 0 & -m l S_{1}-m l S_{2} & \cdots-m l S_{n} \\
0 & (M+n m) r-m l C_{1}-m l C_{2} & \cdots-m l C_{n} \\
-m l S_{1} & -m l r C_{1} & m l^{2} & 0 & \cdots 0 \\
-m l S_{2} & -m l r C_{2} & 0 & m l^{2} & \cdots 0 \\
\vdots & \vdots & \vdots & \vdots & \ddots \\
-m l S_{n} & -m l r C_{n} & 0 & 0 & \cdots m l^{2}
\end{array}\right]
$$

where $S_{i}$ and $C_{i}$ are expressed by

$$
S_{i}=\sin \left(\phi_{i}+\varphi_{i}+\psi\right), \quad C_{i}=\cos \left(\phi_{i}+\varphi_{i}+\psi\right) .
$$


$\mathbf{B}(\mathbf{x})=\left[\dot{r} \dot{\psi} \dot{\phi}_{1} \dot{\phi}_{2} \cdots \dot{\phi}_{n} B_{\dot{r}} B_{\dot{\psi}} B_{\dot{\phi}_{1}} B_{\dot{\phi}_{2}} \cdots B_{\dot{\phi}_{n}}\right]^{T}$, where $B_{\dot{r}}$, and $B_{\dot{\phi}_{i}}$ are expressed by

$$
\begin{aligned}
& B_{\dot{r}}=(M+n m) r(\omega-\dot{\psi})^{2}-k r-c \dot{r}+m \sum_{i=1}^{n}\left[l\left(\dot{\phi}_{i}+\omega\right)^{2} \cos \left(\phi_{i}+\varphi_{i}+\psi\right)\right. \\
& \left.+a \omega^{2} \cos \left(\varphi_{i}+\psi\right)\right]+M \varepsilon \omega^{2} \cos \psi \\
& B_{\dot{\psi}}=2(M+n m) \dot{r}(\omega-\dot{\psi})-c r(\omega-\dot{\psi})-m \sum_{i=1}^{n}\left[l\left(\dot{\phi}_{i}+\omega\right)^{2} \sin \left(\phi_{i}+\varphi_{i}+\psi\right)\right. \\
& \left.+a \omega^{2} \sin \left(\varphi_{i}+\psi\right)\right]-M \varepsilon \omega^{2} \sin \psi, \\
& B_{\dot{\phi}_{i}}=-D \dot{\phi}_{i}-m l r(\omega-\dot{\psi})^{2} \sin \left(\phi_{i}+\varphi_{i}+\psi\right) \\
& -2 m l \dot{r}(\omega-\dot{\psi}) \cos \left(\phi_{i}+\varphi_{i}+\psi\right)-\operatorname{mal}^{2} \sin \phi_{i}, \quad i=1,2, \cdots, n . \\
& \mathbf{C}^{*}=\left[\begin{array}{lllll}
-c & -2(M+n m) \omega r^{*} & 2 m l \omega C_{1}^{*} & 2 m l \omega C_{2}^{*} & \cdots 2 m l \omega C_{n}^{*} \\
2(M+n m) \omega-c r^{*} & \multicolumn{2}{l}{-2 m l \omega S_{1}^{*}-2 m l \omega S_{2}^{*}} & \cdots-2 m l \omega S_{n}^{*} \\
-2 m l \omega C_{1}^{*} & 2 m l \omega r^{*} S_{1}^{*} & -D & 0 & \cdots 0 \\
-2 m l \omega C_{2}^{*} & 2 m l \omega r^{*} S_{2}^{*} & 0 & -D & \cdots 0 \\
\vdots & \vdots & \vdots & \vdots & \ddots \vdots \\
-2 m l \omega C_{n}^{*} & 2 m l \omega r^{*} S_{n}^{*} & 0 & 0 & \cdots-D
\end{array}\right] \text {, } \\
& \mathbf{K}^{*}=\left[\begin{array}{lllll}
-k+(M+n m) \omega^{2} & k_{1} & \multicolumn{3}{c}{-m l \omega^{2} S_{1}^{*}-m l \omega^{2} S_{2}^{*} \cdots-m l \omega^{2} S_{n}^{*}} \\
c \omega & k_{2} & -m l \omega^{2} C_{1}^{*}-m l \omega^{2} C_{2}^{*} \cdots-m l \omega^{2} C_{n}^{*} \\
-m l \omega^{2} S_{1}^{*} & -m l \omega^{2} r^{*} C_{1}^{*} \kappa_{1} & 0 & \cdots 0 \\
-m l \omega^{2} S_{2}^{*} & -m l \omega^{2} r^{*} C_{2}^{*} 0 & \kappa_{2} & \cdots 0 \\
\vdots & \vdots & \vdots & \vdots & \ddots \vdots \\
-m l \omega^{2} S_{n}^{*} & -m l \omega^{2} r^{*} C_{n}^{*} 0 & 0 & \cdots \kappa_{n}
\end{array}\right],
\end{aligned}
$$

where $k_{1}, k_{2}, \kappa_{i}, S_{i}^{*}$ and $C_{i}^{*}$ are expressed by

$$
\begin{aligned}
& k_{1}=-\omega^{2}\left[M \varepsilon \sin \psi^{*}+\sum_{i=1}^{n}\left[m l S_{i}^{*}+m a \sin \left(\varphi_{i}+\psi^{*}\right)\right]\right] \\
& k_{2}=-\omega^{2}\left[M \varepsilon \cos \psi^{*}+\sum_{i=1}^{n}\left[m l C_{i}^{*}+m a \cos \left(\varphi_{i}+\psi^{*}\right)\right]\right] \\
& S_{i}^{*}=\sin \left(\phi_{i}^{*}+\varphi_{i}+\psi^{*}\right), \quad C_{i}^{*}=\cos \left(\phi_{i}^{*}+\varphi_{i}+\psi^{*}\right), \\
& \kappa_{i}=-m l \omega^{2}\left(r^{*} C_{i}^{*}+a \cos \phi_{i}^{*}\right), \quad i=1,2,3, \cdots n . \\
& c_{0}=-\left(\tilde{\varepsilon}^{2}+2 \tilde{m}\right)\left(\tilde{\varepsilon}^{2}-2 \tilde{m}-4 \tilde{m}^{2}\right), \\
& c_{1}=8 \tilde{m}^{2}(1+\tilde{m})(\beta+2 m \beta+2 \zeta), \\
& c_{2}=4\left[-\tilde{\varepsilon}^{4} \tilde{\omega}^{2}+4 \tilde{m}^{4}\left(\tilde{\omega}^{2}+\beta^{2}\right)+2 \tilde{m}^{3}\left(1+3 \tilde{\omega}^{2}+2 \beta^{2}+6 \beta \zeta\right)\right. \\
& \left.+\tilde{m}^{2}\left\{2+\left(2+\tilde{\varepsilon}^{2}\right) \tilde{\omega}^{2}+\beta^{2}+8 \beta \zeta+4 \zeta^{2}\right\}\right]+4 \tilde{a} \tilde{m}\left(1+3 \tilde{m}+2 \tilde{m}^{2}\right) \sqrt{-\tilde{\varepsilon}^{2}+4 \tilde{m}^{2}} \tilde{\omega}^{2},
\end{aligned}
$$




$$
\begin{aligned}
& c_{3}=8 \tilde{m}^{2}\left[2 \zeta\left\{1+(1+4 \tilde{m}) \tilde{\omega}^{2}+\beta^{2}(1+2 \tilde{m})\right\}+\beta\left(2+3 \tilde{m}+2 \tilde{\omega}^{2}+7 \tilde{m} \tilde{\omega}^{2}+6 \tilde{m}^{2} \tilde{\omega}^{2}+4 \zeta^{2}\right)\right] \\
& +4 \tilde{a} \tilde{m}\left[(1+2 \tilde{m})^{2} \beta+2(2+3 \tilde{m}) \zeta\right] \sqrt{-\tilde{\varepsilon}^{2}+4 \tilde{m}^{2}} \tilde{\omega}^{2}, \\
& c_{4}=2\left[-3 \tilde{\varepsilon}^{4} \tilde{\omega}^{4}+16 \tilde{m}^{4} \tilde{\omega}^{2}\left(\tilde{\omega}^{2}+\beta^{2}\right)+4 \tilde{m}^{3}\left\{3 \tilde{\omega}^{4}+2 \beta^{2}+4 \tilde{\omega}^{2}\left(1+\beta^{2}+3 \beta \zeta\right)\right\}\right. \\
& \left.+2 \tilde{m}^{2}\left\{1+\left(1+6 \tilde{\varepsilon}^{2}\right) \tilde{\omega}^{4}+8 \beta \zeta+2 \tilde{\omega}^{2}\left(-1+\beta^{2}+4 \beta \zeta+2 \zeta^{2}\right)+\beta^{2}\left(2+4 \zeta^{2}\right)\right\}\right] \\
& +\tilde{a} \sqrt{-\tilde{\varepsilon}^{2}+4 \tilde{m}^{2}} \tilde{\omega}^{2}\left[24 \tilde{m}^{3} \tilde{\omega}^{2}+4 \tilde{m}^{2}\left(3+7 \tilde{\omega}^{2}+8 \beta \zeta\right)+8 \tilde{m}\left(1+\tilde{\omega}^{2}+2 \beta \zeta+2 \zeta^{2}\right)\right] \\
& +\tilde{a}^{2} \tilde{\omega}^{4}\left[-\tilde{\varepsilon}^{2}-4 \tilde{\varepsilon}^{2} \tilde{m}+4\left(1-\tilde{\varepsilon}^{2}\right) \tilde{m}^{2}+16 \tilde{m}^{3}+16 \tilde{m}^{4}\right] \\
& c_{5}=8 \tilde{m}^{2}\left[6 \tilde{m} \tilde{\omega}^{4} \zeta+2\left(1+\tilde{\omega}^{2}+2 \tilde{m} \tilde{\omega}^{2}\right) \beta^{2} \zeta+\beta+\beta\left(1+5 \tilde{m}+6 \tilde{m}^{2}\right) \tilde{\omega}^{4}+2 \tilde{\omega}^{2}\left(-1+\tilde{m}+2 \zeta^{2}\right)\right] \\
& +4 \tilde{a} \sqrt{-\tilde{\varepsilon}^{2}+4 \tilde{m}^{2}} \tilde{\omega}^{2}\left[8 \tilde{m}^{3} \tilde{\omega}^{2} \beta+4 \tilde{m}^{2}\left(1+2 \tilde{\omega}^{2}\right) \beta+12 \tilde{m}^{2} \tilde{\omega}^{2} \zeta\right. \\
& \left.+4 m\left(1+\omega^{2}\right) \zeta+2 m \beta\left(1+\omega^{2}+2 \zeta^{2}\right)\right]+4 \tilde{a}^{2} \tilde{\omega}^{4}\left(-\tilde{\varepsilon}^{2}-2 \tilde{\varepsilon}^{2} \tilde{m}+4 \tilde{m}^{2}+8 \tilde{m}^{3}\right) \zeta, \\
& c_{6}=4 \tilde{\omega}^{2}\left[-\tilde{\varepsilon}^{4} \tilde{\omega}^{4}+4 \tilde{m}^{4} \tilde{\omega}^{2}\left(\tilde{\omega}^{2}+\beta^{2}\right)+2 \tilde{m}^{3}\left\{\tilde{\omega}^{4}-2 \beta^{2}+\tilde{\omega}^{2}\left(-1+2 \beta^{2}+6 \beta \zeta\right)\right\}\right] \\
& +4 \tilde{m}^{2}\left[4 \tilde{\varepsilon}^{2} \tilde{\omega}^{6}+\beta^{2}\left\{1+\tilde{\omega}^{4}+\tilde{\omega}^{2}\left(-2+4 \zeta^{2}\right)\right\}\right] \\
& +4 \tilde{a} \tilde{m} \sqrt{-\tilde{\varepsilon}^{2}+4 \tilde{m}^{2}} \tilde{\omega}^{2}\left[1+\left(1+5 \tilde{m}+6 \tilde{m}^{2}\right) \tilde{\omega}^{4}+4 \beta \zeta\right. \\
& \left.+2 \tilde{\omega}^{2}\left(-1+\tilde{m}+2 \beta \zeta+4 \tilde{m} \beta \zeta+2 \zeta^{2}\right)\right] \\
& -2 \tilde{a}^{2}\left(\tilde{\varepsilon}^{2}-4 \tilde{m}^{2}\right) \tilde{\omega}^{4}\left[1+\tilde{\omega}^{2}+4 \tilde{m}^{2} \tilde{\omega}^{2}+\tilde{m}\left(2+4 \tilde{\omega}^{2}\right)+2 \zeta^{2}\right], \\
& c_{7}=8 \tilde{m}^{3} \tilde{\omega}^{4}\left[-1+(1+2 \tilde{m}) \tilde{\omega}^{2}\right] \beta+4 \tilde{a} \tilde{m} \sqrt{-\tilde{\varepsilon}^{2}+4 \tilde{m}^{2}} \tilde{\omega}^{2}\left[6 \tilde{m} \tilde{\omega}^{4} \zeta\right. \\
& \left.+\beta\left\{1+(1+2 \tilde{m})^{2} \tilde{\omega}^{4}-2 \tilde{\omega}^{2}\left(1+2 \tilde{m}-2 \zeta^{2}\right)\right\}\right]-4 \tilde{a}^{2}\left(\tilde{\varepsilon}^{2}-4 \tilde{m}^{2}\right) \tilde{\omega}^{4}\left[1+(1+2 \tilde{m}) \tilde{\omega}^{2}\right] \zeta, \\
& c_{8}=-\tilde{\varepsilon}^{2}\left(\tilde{\varepsilon}^{2}-4 \tilde{m}^{2}\right) \tilde{\omega}^{8}+4 \tilde{a} \tilde{m}^{2} \sqrt{-\tilde{\varepsilon}^{2}+4 \tilde{m}^{2}} \tilde{\omega}^{6}\left[-1+(1+2 \tilde{m}) \tilde{\omega}^{2}\right] \\
& -\tilde{a}^{2}\left(\tilde{\varepsilon}^{2}-4 \tilde{m}^{2}\right) \tilde{\omega}^{4}\left[1+(1+2 \tilde{m})^{2} \tilde{\omega}^{4}-2 \tilde{\omega}^{2}\left(1+2 \tilde{m}-2 \zeta^{2}\right)\right] .
\end{aligned}
$$

\section{References}

[1] J.D. Alexander, An Automatic Dynamic Balancer, Proceedings for the Second Southeastern Conference 2 (1964), 415-426.

[2] J.W. Cade, Self-compensating in Rotating Mechanisms, Design News (1965), 234-239.

[3] J. Lee, An analytical Study of Self-compensating Dynamic Balancer with Damping Fluid and Ball, Shock and Vibration 2(1) (1995), 59-67.

[4] J. Chung, Dynamic Analysis of an Automatic Dynamic Balancer for Rotating Mechanisms, Journal of Sound and Vibration 228(5) (1999), 1035-1056.

[5] C.H. Hwang, Dynamic Analysis of an Automatic Dynamic ball Balancer with Double Races, JSME International Journal 42(2) (1999), $265-272$.

[6] E.H. Cho, Dynamic Analysis of an Automatic Ball Balancer with Triple Races, Proceedings of 2002 KSME Meeting 42(4) (2002), 764-774.

[7] W.-Y. Huang and C.-P. Chao, The Application of Ball-Type Balancers for Radial Vibration of High-Speed Optic Disk Drives, Journal of Sound and Vibration 250(3) (2002), 415-430.

[8] S. Bae, Dynamic Analysis of an Automatic Washing Machine with a Hydraulic Balancer, Journal of Sound and Vibration 257(1) (2002), 3-18.

[9] J. Sohn, Application of Automatic Dynamic Banalcing System to the Optical Disk Drive, Proceeding of Inter $\cdot$ noise $200063685-3688$. 

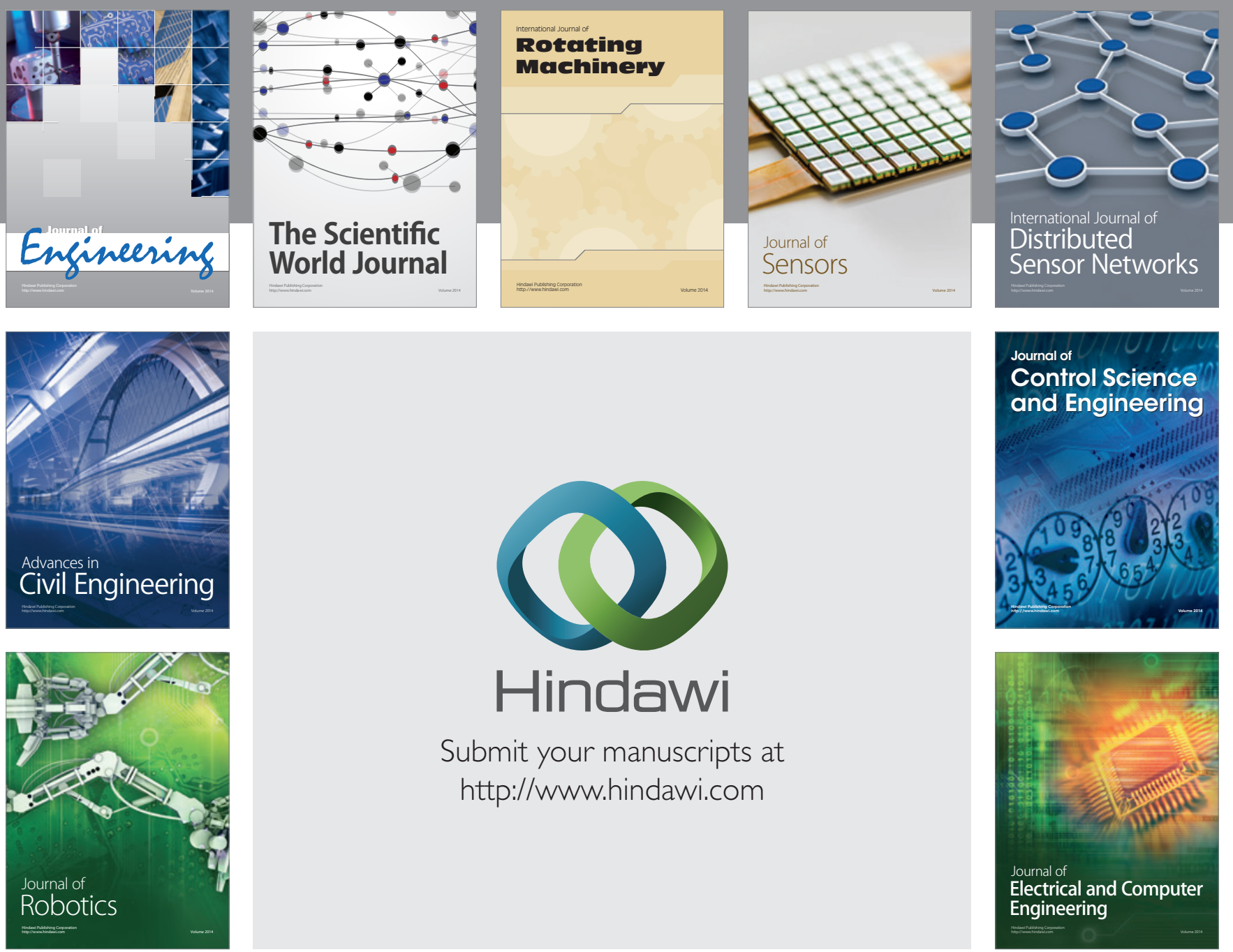

Submit your manuscripts at

http://www.hindawi.com
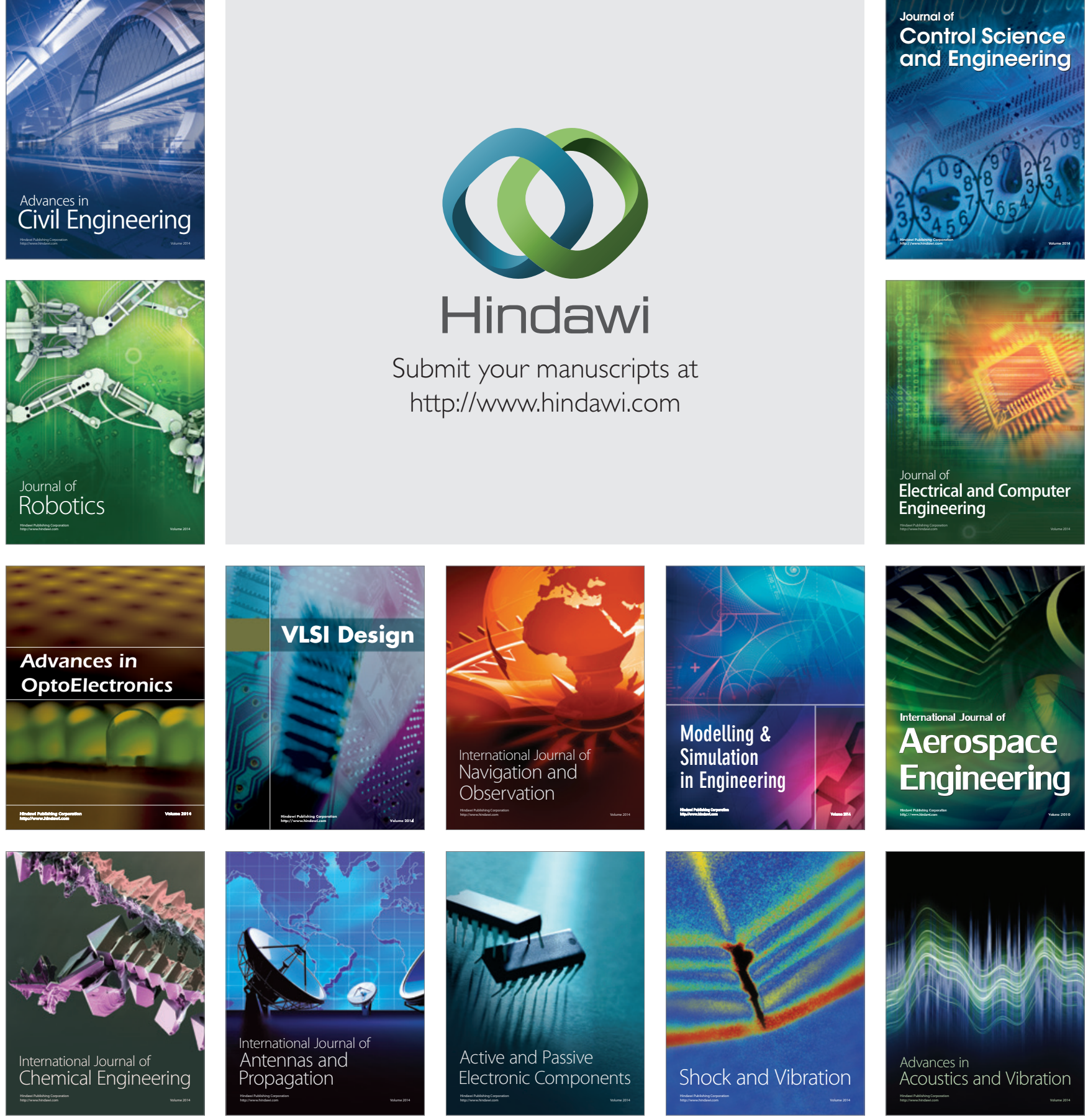